\title{
Depth-of-field effects in wiggler radiation sources: Geometrical versus wave optics
}

\author{
Richard P. Walker" \\ Diamond Light Source, Oxfordshire OX11 ODE, United Kingdom
}

(Received 14 December 2016; published 8 February 2017)

\begin{abstract}
A detailed analysis is carried out of the optical properties of synchrotron radiation emitted by multipole wigglers, concentrating on the effective source size and brightness and the so-called "depth of field" effects, concerning which there has been some controversy in the literature. By comparing calculations made with both geometrical optics and wave optics methods we demonstrate that the two approaches are not at variance, and that the wave optics results tend towards those of geometrical optics under well-defined conditions.
\end{abstract}

DOI: 10.1103/PhysRevAccelBeams.20.020703

\section{INTRODUCTION}

The optical properties of synchrotron radiation sources have been studied for many years. Sabersky investigated the imaging of bending magnet radiation using geometrical optics and phase space techniques and pointed out that because of the length of arc seen by the observer the image is subject to depth of field errors [1]. Green continued this work, producing expressions also for the brightness of the radiation, i.e. the photon flux density in four-dimensional phase space [2]. Later Coisson et al. extended the analysis to wiggler sources and showed that projecting the radiation to the center of the device produces a complex pattern and also that there is a significant increase in source size as a function of angular acceptance due to depth of field effects [3]. The authors also considered the conditions under which interference effects that are present in a periodic magnetic field device can be neglected.

Further studies of depth of field effects in wigglers, using the same geometrical optics approach, were reported in Refs. [4-8]. The effects are well known, namely the increase in horizontal source size as a function of off-axis viewing angle, or angular acceptance, as well as the decrease in brightness resulting in a less than linear dependence on device length. Unfortunately, few direct measurements of such effects have been reported in the literature, however the few that exist do show agreement with expectations $[9,10]$.

Studies of the properties of radiation emitted by undulators developed in parallel to that of wigglers. Although the spectral and angular properties of undulators were well

\footnotetext{
r.p.walker@diamond.ac.uk
}

Published by the American Physical Society under the terms of the Creative Commons Attribution 4.0 International license. Further distribution of this work must maintain attribution to the author(s) and the published article's title, journal citation, and DOI. understood from the beginning [11,12], difficulties arose in addressing the source size and brightness and there was some confusion over whether depth of field (i.e. geometrical optics) effects should be treated separately from diffraction (i.e. wave optics) effects [13,14]. Later Kim [15] and Coisson and Walker [16] showed that the effects are in fact the same and should not be added. At the same time a more rigorous approach to calculating brightness was proposed based on the Wigner distribution [15,16], which has been followed by several papers on the subject [17-24].

Although the wave optics approach to undulator sources is well developed and accepted, the application to wiggler sources is less advanced, mainly as a result of the computational difficulty. One of the first studies of wigglers using wave optics was Ref. [21] where it was shown that "the intensity distributions are considerably affected by the 'depth of field' effect." More recently however Geloni et al. [24] also examined wiggler radiation using a wave optics calculation but their surprising conclusion that "according to electrodynamics, depth-of-field effects do not exist," and that they are a "misconception," is clearly at odds with previous work. Stimulated by this and other similarly robust and challenging assertions, we present in this article a more complete comparison of the wave optics and geometrical optics methods in relation to wiggler sources and we show categorically for the first time that under appropriate conditions the two methods are indeed consistent.

Section II first outlines the geometrical optics model, with some new aspects compared to previous work. Section III outlines a simplified wave optics approach that has been developed in order to allow different features to be more easily incorporated compared to existing codes. Section IV then makes a detailed comparison of the two methods, for both the source size distribution and radiation brightness, and finally Secs. V and VI discuss the results and present conclusions. In order to be able to make a direct comparison with Ref. [24], most of the calculations 
TABLE I. Wiggler, electron and photon parameters used for simulations, unless othewise stated, following Ref. [24].

Period length $\left(\lambda_{o}\right)$

Number of periods $(N)$

Length $(L)$

Field amplitude $\left(B_{o}\right)$

Deflection parameter $(K)$

Electron energy $(E)$

Beam current $(I)$

Horizontal emittance $\left(\varepsilon_{x}\right)$

Vertical emittance $\left(\varepsilon_{y}\right)$

Beta functions $\left(\beta_{x}, \beta_{y}\right)$

Relative energy spread $\left(\sigma_{\varepsilon}\right)$

Critical photon energy

Photon energy for calculations

Harmonic number $(n)$

reported here will use the same wiggler and electron beam parameters which are given in Table I.

\section{GEOMETRICAL OPTICS MODEL}

\section{A. Expressions for brightness and spatial distribution of intensity}

A geometrical optics model for a wiggler was described in [8] so will not be repeated in full here. We assume a plane periodic wiggler magnet with sinusoidal vertical field distribution of amplitude $B_{o}$ and period length $\lambda_{o}$ leading to the following trajectory in the horizontal plane:

$x_{c}(s)=a \sin (k s)=\frac{K}{\gamma} \frac{\lambda_{o}}{2 \pi} \sin (k s), \quad x_{c}^{\prime}(s)=\frac{K}{\gamma} \cos (k s)$,

where $k=2 \pi / \lambda_{o}, K=e B_{o} \lambda_{o} / 2 \pi m c$. If a particular electron has offset and angle at the center of the device given by $x_{o}, x_{o}^{\prime}, y_{o}, y_{o}^{\prime}$, and emits photons at position $s$ at angle $\theta_{x}, \theta_{y}$, with respect to the tangent to the trajectory at that point, the projection of this ray to the center of the device, $s=0$, gives the following phase space coordinates:

$x=x_{o}+x_{c}(s)-x_{c}^{\prime}(s) s-\theta_{x} s \quad x^{\prime}=x_{o}^{\prime}+x_{c}^{\prime}(s)+\theta_{x}$

$y=y_{o}-\theta_{y} s \quad y^{\prime}=y_{o}^{\prime}+\theta_{y}$.

In the horizontal plane terms in $\theta_{x}$ can be neglected compared to those in $x_{c}^{\prime}$ since we assume $K \gg 1$. It follows that the photon flux density in phase space at the center of the wiggler is then

$$
\begin{aligned}
\frac{d^{4} \Phi\left(x, x^{\prime}, y, y^{\prime}\right)}{d x d x^{\prime} d y d y^{\prime}}= & \int_{-L / 2}^{L / 2} \frac{d s}{\rho(s)} \int_{-\infty}^{\infty} d \theta_{y} \frac{d^{2} \Phi\left(s, \theta_{y}\right)}{d \Omega} \\
& \times P\left(x_{o}\right) P\left(x_{o}^{\prime}\right) P\left(y_{o}\right) P\left(y_{o}^{\prime}\right),
\end{aligned}
$$

where $d^{2} \Phi / d \Omega$ is the angular and spectral flux density, i.e. photons/(s unit bandwidth unit solid angle), for a single bending magnet which is given by the well-known expression, e.g. Ref. [8]:

$$
\begin{aligned}
\frac{d^{2} \Phi}{d \Omega} & =\frac{3 \alpha}{4 \pi^{2}} \frac{I}{e} \gamma^{2}\left(\frac{\omega}{\omega_{c}}\right)^{2} \\
& \times\left[\left(1+X^{2}\right)^{2} K_{2 / 3}^{2}(\xi)+X^{2}\left(1+X^{2}\right) K_{1 / 3}^{2}(\xi)\right]
\end{aligned}
$$

where $X=\gamma \theta_{y}, \xi=0.5\left(\omega / \omega_{c}\right)\left(1+X^{2}\right)^{3 / 2}, I$ is the beam current and $\omega_{c}$ is the critical frequency. The functions $P$ are Gaussian distributions for the electron beam size and divergence in the horizontal and vertical planes [i.e. $\left.P(z)=\exp -\left(z^{2} / 2 \sigma_{z}^{2}\right) / \sqrt{2 \pi} \sigma_{z}\right]$ with rms values given by $\sigma_{x}=\sqrt{\varepsilon_{x} \beta_{x}}, \sigma_{x^{\prime}}=\sqrt{\varepsilon_{x} / \beta_{x}}$ etc., where $\varepsilon_{x}, \varepsilon_{y}$ are the electron beam emittances, and we assume for simplicity that the wiggler is located at a symmetry point in the ring.

Proceeding as in [8], by assuming the horizontal acceptance angle is relatively small so that the emission occurs essentially close to the pole centers $s=s_{i}$ and integrating over the vertical emission angle assuming a Gaussian distribution with rms value $\sigma_{\psi}$, allows the following expression to be derived for the flux density in phase space, i.e. the brightness, as a summation over the number of poles:

$$
\begin{aligned}
\mathscr{B}= & \frac{d^{4} \Phi\left(x, x^{\prime}, y, y^{\prime}\right)}{d x d x^{\prime} d y d y^{\prime}} \\
= & \left.\frac{d^{2} \Phi}{d \Omega}\right|_{\theta_{y}=0} \sqrt{2 \pi} \sigma_{\psi} \sum_{i=1}^{N \text { poles }} \frac{1}{\sqrt{2 \pi}\left(\sigma_{x}^{2}+\sigma_{x \prime}^{2} s_{i}^{2}\right)^{1 / 2}} \\
& \times \exp \left(-\frac{\left(x \pm a+x^{\prime} s_{i}\right)^{2}}{2\left(\sigma_{x}^{2}+\sigma_{x \prime}^{2} s_{i}^{2}\right)}\right) \frac{1}{2 \pi \varepsilon^{1 / 2}} \\
& \times \exp \left(-\frac{\gamma y^{2}+2 \alpha y y^{\prime}+\beta y^{\prime 2}}{2 \varepsilon}\right)
\end{aligned}
$$

where

$$
\begin{aligned}
& \alpha=s_{i} \sigma_{\psi}^{2} \quad \beta=\sigma_{y}^{2}+s_{i}^{2} \sigma_{\psi}^{2} \quad \gamma=\sigma_{y^{\prime}}^{2}+\sigma_{\psi}^{2} \\
& \varepsilon=s_{i}^{2} \sigma_{y \prime}^{2} \sigma_{\psi}^{2}+\sigma_{y}^{2} \sigma_{\psi}^{2}+\sigma_{y}^{2} \sigma_{y^{\prime}}^{2} .
\end{aligned}
$$

In the following we use the standard definition for $\sigma_{\psi}$ which gives the correct relation between the peak and integrated spectral flux density:

$$
\sqrt{2 \pi} \sigma_{\psi}=\frac{d \Phi}{d \theta_{x}} /\left.\frac{d^{2} \Phi}{d \Omega}\right|_{\theta_{y}=0} .
$$

It follows that the expression for the central brightness of the radiation is given by the following:

$$
\begin{aligned}
\mathscr{B}_{o}= & \frac{d^{4} \Phi(0,0,0,0)}{d x d x \prime d y d y \prime}=\frac{d \Phi}{d \theta_{x}} \sum_{i=1}^{N_{\text {poles }}} \frac{1}{\sqrt{2 \pi}\left(\sigma_{x}^{2}+\sigma_{x \prime}^{2} s_{i}^{2}\right)^{1 / 2}} \\
& \times \exp \left(-\frac{a^{2}}{2\left(\sigma_{x}^{2}+\sigma_{x \prime}^{2} s_{i}^{2}\right)}\right) \frac{1}{2 \pi \varepsilon^{1 / 2}} .
\end{aligned}
$$


An equivelant expresion was given by Kim [4]. This can be further approximated in the limit $L \leq 4 \beta_{y}, L \leq 2 \beta_{x}, a \leq$ $\sigma_{x}, \sigma_{y^{\prime}} \leq \sigma_{\psi} / 2$ as follows [8]:

$$
\mathscr{B}_{o} \simeq \frac{d \Phi}{d \theta_{x}} \frac{N_{\text {poles }}}{(2 \pi)^{3 / 2}\left(\sigma_{x}^{2}+a^{2}+\sigma_{x^{\prime}}^{2} L^{2} / 12\right)^{1 / 2}\left(\sigma_{y}^{2}+\sigma_{y^{\prime}}^{2} L^{2} / 12\right)^{1 / 2}\left(\sigma_{y \prime}^{2}+\sigma_{\psi}^{2}\right)^{1 / 2}} .
$$

As well as examining the validity of these expressions for brightness, we are also particularly interested in the source size distribution which can be approximated as follows:

$$
\frac{d^{2} \Phi(x, y)}{d x d y}=\int_{-\Theta_{x}}^{\Theta_{x}} \int_{-\Theta_{y}}^{\Theta_{y}} \mathscr{B}\left(x, x^{\prime}, y, y^{\prime}\right) d x^{\prime} d y^{\prime}
$$

where $\pm \Theta_{x}, \pm \Theta_{y}$ are the horizontal and vertical acceptance angles.

A relevant point that has not been discussed in previous work is the fact that the horizontal source size distribution depends on how the vertical plane is treated. There are several different ways of proceeding, as discussed below.

\section{Integrate over all vertical angles and all vertical positions}

In this case we have

$$
\begin{aligned}
\frac{d \Phi(x)}{d x}= & \frac{d \Phi}{d \theta_{x}} \sum_{i=1}^{N \text { poles }} \int_{-\Theta_{x}}^{\Theta_{x}} d x^{\prime} \frac{1}{\sqrt{2 \pi}\left(\sigma_{x}^{2}+\sigma_{x^{\prime}}^{2} s_{i}^{2}\right)^{1 / 2}} \\
& \times \exp \left(-\frac{\left(x \pm a+x^{\prime} s_{i}\right)^{2}}{2\left(\sigma_{x}^{2}+\sigma_{x^{\prime}}^{2} s_{i}^{2}\right)}\right)
\end{aligned}
$$

Further analysis leads to estimates for the rms of the distribution at a given angle $\theta_{x}$ :

$$
\Sigma_{x}=\left(\sigma_{x}^{2}+a^{2}+L^{2} \sigma_{x \prime}^{2} / 12+L^{2} \theta_{x}^{2} / 12\right)^{1 / 2}
$$

and when integrated over the angle $\pm \Theta_{x}$ :

$$
\Sigma_{x}=\left(\sigma_{x}^{2}+a^{2}+L^{2} \sigma_{x \prime}^{2} / 12+L^{2} \Theta_{x}^{2} / 36\right)^{1 / 2}
$$

Although these latter two equations approximate Eq. (5) well, it is well known that the distribution function Eq. (5) can be highly non-Gaussian, with significantly wide "tails" such that the rms value is not a very satisfactory measure of the overall source size and so must be used with caution.

\section{Integrate over a given vertical angular acceptance, horizontal cut at $y=0$}

In this case we have

$$
\begin{aligned}
\left.\frac{d^{2} \Phi(x)}{d x d y}\right|_{y=0}= & \frac{d \Phi}{d \theta_{x}} \sum_{i=1}^{N \text { poles }} \int_{-\Theta_{x}}^{\Theta_{x}} \int_{\Theta_{y}}^{\Theta_{y}} d x^{\prime} d y^{\prime} \frac{1}{\sqrt{2 \pi}\left(\sigma_{x}^{2}+\sigma_{x \prime}^{2} s_{i}^{2}\right)^{1 / 2}} \\
& \times \exp \left(-\frac{\left(x \pm a+x^{\prime} s_{i}\right)^{2}}{2\left(\sigma_{x}^{2}+\sigma_{x \prime}^{2} s_{i}^{2}\right)}\right) \frac{1}{2 \pi \varepsilon^{1 / 2}} \exp \left(-\frac{\beta y^{\prime 2}}{2 \varepsilon}\right) .
\end{aligned}
$$

It is clear that the additional vertical terms in the summation compared to Eq. (5) have a different dependence on $s_{i}$, and so will influence the depth-of-field effects.

\section{Integrate over a given vertical angular acceptance, integrate over all vertical positions}

In this case we have

$$
\begin{aligned}
\frac{d \Phi(x)}{d x}= & \frac{d \Phi}{d \theta_{x}} \sum_{i} \int_{-\Theta_{x}}^{\Theta_{x}} d x^{\prime} \int_{-\Theta_{y}}^{\Theta_{y}} d y^{\prime} \frac{1}{\sqrt{2 \pi}\left(\sigma_{x}^{2}+\sigma_{x \prime}^{2} s_{i}^{2}\right)^{1 / 2}} \\
& \times \exp \left(-\frac{\left(x \pm a+x^{\prime} s_{i}\right)^{2}}{2\left(\sigma_{x}^{2}+\sigma_{x \prime}^{2} s_{i}^{2}\right)}\right) \frac{1}{\sqrt{2 \pi} \gamma^{1 / 2}} \exp \left(-\frac{y^{\prime 2}}{2 \gamma}\right)
\end{aligned}
$$

which because $\gamma$ is independent of $s$, leads to the same horizontal distribution, in relative units, as case 1 above.

\section{B. Comparison of the geometrical optics model with measurements}

Berman and Yin measured the horizontal source size of nonmonochromatized radiation as a function of horizontal angle in the X25 wiggler [10]. Figure 1 reproduces this data which shows clear evidence of a broadening of the horizontal size as a function of the off-axis viewing angle and also that the geometrical optics model is in good agreement with the measured data. In this case the geometry used for the measurements corresponds to case 3 above, and hence Eq. (5) has been used for the analysis. The source size is defined as the region bounded by the $10 \%$ and $90 \%$ boundaries of the integrated intensity profile produced by scanning a slit across the beam, as in Ref. [10]. It can also be seen that the approximation Eq. (6), when scaled appropriately assuming a Gaussian intensity 


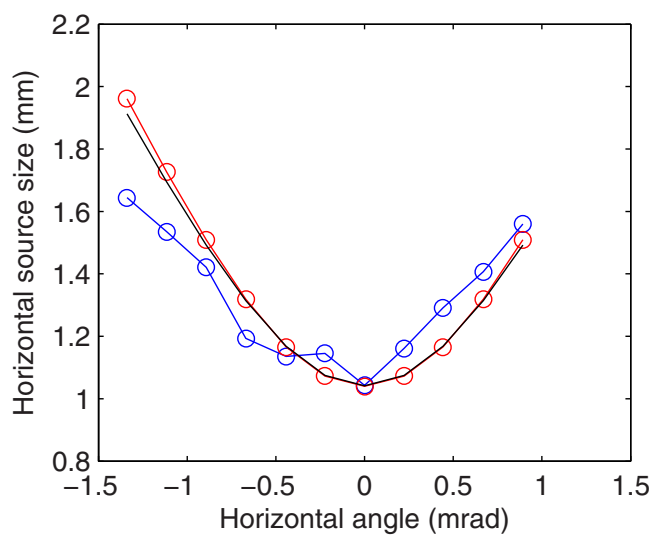

FIG. 1. Horizontal source size $(10 \%-90 \%$ of integrated intensity) as a function of off-axis horizontal angle for the NSLS X25 wiggler; blue — measured data [10]; red—geometrical optics model Eq. (5); black-Eq. (6), rms x 2.563.

distribution, is also in good agreement, reflecting the fact that the wiggler is relatively short $(1.62 \mathrm{~m})$ and hence the intensity distribution still approximates a Gaussian function.

\section{SIMPLIFIED WAVE OPTICS MODEL}

\section{A. Angular and spatial distributions}

In this section we introduce a simplified wave optics model based on the "dipole series model" [25], in which a periodic magnet is represented by a series of dipole magnet sources with the appropriate phase relationship between them, which has been shown in Refs. [25,26] to be sufficiently accurate compared to more exact methods of calculation. The advantages of this approach are first that it allows a simple way of identifying the physical cause for the various features that are observed, and second that it is computationally efficient and so allows other crucial aspects to be included relatively easily, particular energy spread and multiple harmonics.

We start by rewriting the expression for the spectral and angular flux density from a single bending magnet, Eq. (1), as follows:

$$
\frac{d^{2} \Phi}{d \Omega}=\alpha \frac{I}{e}\left(\frac{2 c \varepsilon_{o}}{e}\right)^{2}\left[\left|E_{x}\right|^{2},\left|E_{y}\right|^{2}\right],
$$

where $E_{x}, E_{y}$ are the electric fields in the angular representation [18] polarized in the horizontal and vertical planes respectively, which are given by

$$
\begin{aligned}
{\left[E_{x o}(\bar{\theta}), E_{y o}(\bar{\theta})\right]=} & \frac{\sqrt{3} e \gamma}{4 \pi \varepsilon_{o} c}\left(\frac{\omega}{\omega_{c}}\right)\left[-(1+X)^{2} K_{2 / 3}(\xi),\right. \\
& \left.i X\left(1+X^{2}\right)^{1 / 2} K_{1 / 3}(\xi)\right] .
\end{aligned}
$$

The "dipole series model" [25] is obtained by adding together the amplitudes from a series of such dipoles, with appropriate phase factors $e^{i \delta}$, where $\delta=\omega(t-\bar{\theta} \cdot \bar{r} / c), \bar{r}$ is the electron trajectory and $\bar{\theta}=\left[\theta_{x}, \theta_{y}, 1-\left(\theta_{x}^{2}+\theta_{y}^{2}\right) / 2\right]$ is the direction of emission:

$$
\begin{aligned}
{\left[E_{x}(\bar{\theta}), E_{y}(\bar{\theta})\right]=} & {\left[\left(e^{i \delta_{1}}-e^{i \delta_{2}}\right) E_{x o},\left(e^{i \delta_{1}}+e^{i \delta_{2}}\right) E_{y o}\right] } \\
& \times \sum_{m=-N / 2}^{N / 2-1} e^{i 2 \pi m \omega / \omega_{1}} .
\end{aligned}
$$

The two phase factors involving $\delta_{1}, \delta_{2}$ correspond to the two poles within one period, while the summation is over the $N$ periods in the device. The phase factor when the electron is emitting radiation at horizontal angle $\alpha=\gamma \theta_{x} / K$ is in general given by

$$
\delta=\frac{\omega}{\omega_{1}}\left(\frac{2 \pi s}{\lambda_{o}}-\frac{2 \alpha K^{2}}{A} \sin (k s)+\frac{K^{2}}{4 A} \sin (2 k s)\right),
$$

where $A=1+K^{2} / 2+\gamma^{2} \theta^{2}$ and $\theta^{2}=\theta_{x}^{2}+\theta_{y}^{2}$. This differs from Ref. [25] only in the choice of a trajectory based on a sine function rather than cosine, which guarantees that $\delta=0$ at the center of the device, $s=0$.

The two points of emission corresponding to angle $\alpha$ are $k s_{1}=\cos ^{-1}(\alpha)$ and $k s_{2}=2 \pi-\cos ^{-1}(\alpha)$. Inserting these, summing up the geometric series of phase factors and simplifying yields the following:

$\left[E_{x}(\bar{\theta}), E_{y}(\bar{\theta})\right]=2\left[i E_{x o} \sin (\Delta), E_{y o} \cos (\Delta)\right] \frac{\sin N \pi \omega / \omega_{1}}{\sin \pi \omega / \omega_{1}}$,

where $\Delta=\frac{\omega}{\omega_{1}}\left(-\pi+\cos ^{-1}(\alpha)-\frac{3 K^{2}}{2 A} \alpha\left(1-\alpha^{2}\right)^{1 / 2}\right), \omega$ is the frequency of interest, chosen to correspond to the $n$th harmonic on-axis: $\omega=n\left[4 \pi c \gamma^{2} / \lambda_{o}\left(1+K^{2} / 2\right)\right]$, and $\omega_{1}$ is the fundamental at the angle of emission, $\theta: \omega_{1}=$ $\left[4 \pi c \gamma^{2} / \lambda_{o}\left(1+K^{2} / 2+\gamma^{2} \theta^{2}\right)\right]$.

The spatial distribution of radiation amplitude at the center of the wiggler can then be obtained from this as follows:

$E_{x}(x, y)=\frac{1}{\lambda} \int_{-\Theta_{x}}^{\Theta_{x}} \int_{-\Theta_{y}}^{\Theta_{y}} E_{x}\left(\theta_{x}, \theta_{y}\right) \exp \left(i \frac{2 \pi}{\lambda}\left(\theta_{x} x+\theta_{y} y\right)\right) d \theta_{x} d \theta_{y}$

and similarly for $E_{y}$, where again $\pm \Theta_{x}, \pm \Theta_{y}$ are the horizontal and vertical acceptance angles. This of course is an approximation, the full calculation carried out in Ref. [24] involves calculating the brightness function in the center of the wiggler, transporting it downstream to an aperture, applying the aperture then simulating a perfect lens to form a $1: 1$ image of the source. The approximation used here is however valid when the aperture is sufficiently 


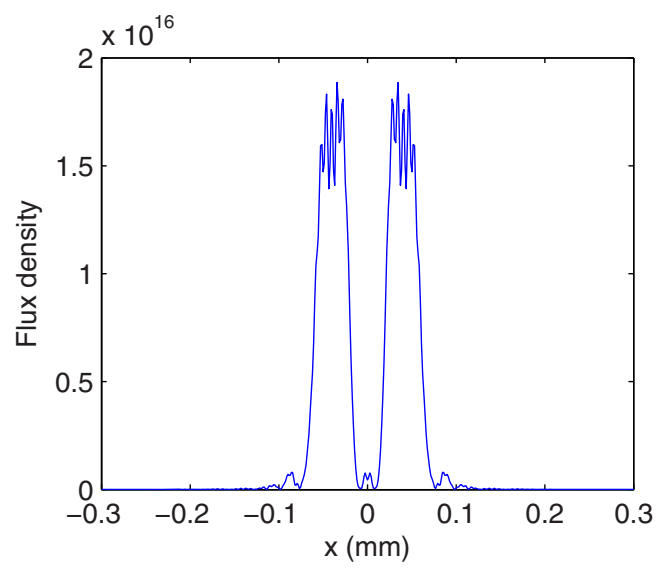

position or angle by the same amount. The electron beam divergence can therefore be taken into account in this simplified model by summing the intensities from individual electrons, with angular limits modified according to the angle of the electron passing through the wiggler, $x_{o}^{\prime}, y_{o}^{\prime}$ :

$$
\begin{aligned}
\frac{d^{2} \Phi}{d x d y}(x, y)= & \alpha \frac{I}{e}\left(\frac{2 c \varepsilon_{o}}{e}\right)^{2} \int_{-\infty}^{\infty} \int_{-\infty}^{\infty} P\left(x_{o}^{\prime}\right) P\left(y_{o}^{\prime}\right) d x_{o}^{\prime} d y_{o}^{\prime} \\
& \times \mid \frac{1}{\lambda} \int_{-\Theta_{x}-x_{o}^{\prime}}^{\Theta_{x}-x_{o}^{\prime}} \int_{\Theta_{y}-y_{o}^{\prime}}^{\Theta_{y}-y_{o}^{\prime}} E_{x}\left(\theta_{x}, \theta_{y}\right) \\
& \times\left.\exp \left(i \frac{2 \pi}{\lambda}\left(\theta_{x} x+\theta_{y} y\right)\right) d \theta_{x} d \theta_{y}\right|^{2} .
\end{aligned}
$$

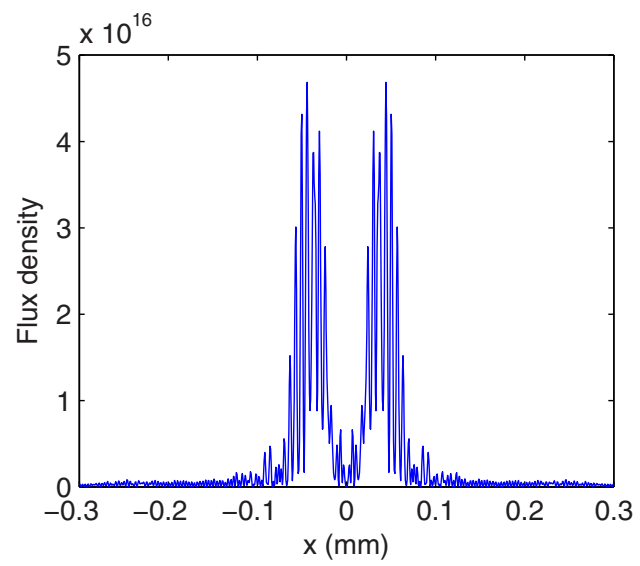

FIG. 2. Spatial flux density, photons/(s $\mathrm{mm}^{2} 0.1 \%$ bandwidth), in the center of the wiggler, horizontal cut at $y=0$, with zero emittance and energy spread. Vertical aperture $\pm 0.125 \mathrm{mrad}$, horizontal aperture (upper) $\pm 0.125 \mathrm{mrad}$, (lower) $\pm 0.25 \mathrm{mrad}$.

large compared to the source size, and that the distance between source and aperture is large compared to the length of the wiggler, both of which apply in this case.

The spectral intensity distribution, photons $/\left(\mathrm{s} \mathrm{mm}^{2}\right.$ unit bandwidth), in the center of the wiggler is then obtained as follows:

$$
\frac{d^{2} \Phi}{d x d y}(x, y)=\alpha \frac{I}{e}\left(\frac{2 c \varepsilon_{o}}{e}\right)^{2}\left[\left|E_{x}(x, y)\right|^{2}+\left|E_{y}(x, y)\right|^{2}\right]
$$

although in all cases discussed here the vertical component can be neglected.

We first show that this simplified wave optics model can give accurate results in the present case. Figure 2 shows the spatial distribution in the center of the representative wiggler (Table I) for two different apertures, in the absence of electron beam emittance and energy spread. The agreement with Fig. 11 of Ref. [24] is excellent, both qualitatively and quantitatively.

The effect of a displacement or angular offset of the electron beam is simply to move the radiation pattern in

Finally this distribution is then convoluted with the electron beam size distribution:

$$
\begin{aligned}
\frac{d^{2} \Phi}{d x d y}(x, y)= & \int_{-\infty}^{\infty} \int_{-\infty}^{\infty} \frac{d^{2} \Phi}{d x d y}\left(x-x_{o}, y-y_{o}\right) \\
& \times P\left(x_{o}\right) P\left(y_{o}\right) d x d y .
\end{aligned}
$$

\section{B. Brightness}

As mentioned in the Introduction, the most accepted definition of brightness using the wave optics model is based on the Wigner distribution [15-24]:

$$
\begin{aligned}
\mathscr{B}_{x}\left(x, x^{\prime}, y, y^{\prime}\right)= & \frac{2 \varepsilon_{o} c}{h} \frac{1}{\lambda^{2}} \frac{I}{e} \int_{-\infty}^{\infty} \int_{-\infty}^{\infty} E_{x}^{*}\left(x^{\prime}+\frac{\theta_{x}}{2}, y^{\prime}+\frac{\theta_{y}}{2}\right) \\
& \times E_{x}\left(x^{\prime}-\frac{\theta_{x}}{2}, y^{\prime}-\frac{\theta_{y}}{2}\right) \\
& \times \exp \left(-i \frac{2 \pi}{\lambda}\left(x \theta_{x}+y \theta_{y}\right)\right) d \theta_{x} d \theta_{y} .
\end{aligned}
$$

Here $\mathscr{B}_{x}$ refers to the brightness of the radiation polarized in the horizontal plane produced from the horizontal electric field component $E_{x}$ as given in the section above. A similar expression exists for the vertically polarized component, however in what follows this can be ignored as it is always much smaller than the horizontal component. We will therefore drop the subscript $x$.

We first show the validity of the current method by comparing the central brightness calculated with Eq. (13) with the results from the well-known code SPECTRA [27] under the same conditions. Figure 3 shows the brightness as a function of harmonic number for the wiggler of Table I and it can be seen that there is very good agreement between the two codes, the only small discrepancy appearing at low harmonic numbers where the dipole series model 


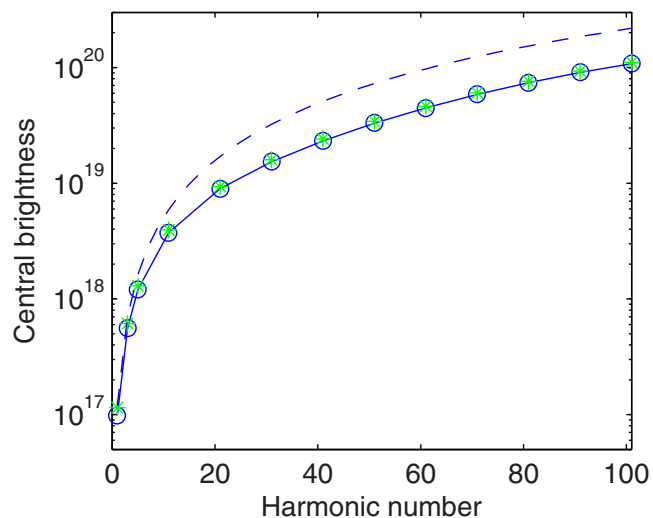

FIG. 3. Central brightness, photons/(s $\mathrm{mm}^{2} \mathrm{mrad}^{2} 0.1 \%$ bandwidth), as a function of harmonic number, with zero emittance, zero energy spread; blue circles-Eq. (13); green asterisksresults obtained using SPECTRA; dashed line-Eq. (14).

is known to be less accurate [25]. In passing, we also compare these results to the analytic result which can be derived from Eq. (13), e.g. see [18]:

$$
\mathscr{B}_{o}=\frac{\Phi_{n}}{2} \frac{4}{\lambda^{2}},
$$

where $\Phi_{n}$ is the total flux in the "central cone," which in practical units, photons/(s $0.1 \%$ bandwidth), is given, for $n=$ odd, by the well-known formula e.g. [18]:

$$
\begin{aligned}
\Phi_{n}= & 1.43110^{14} N I \frac{n K^{2}}{\left(1+K^{2} / 2\right)} \\
& \times\left\{J_{\frac{n-1}{2}}\left[\frac{n K^{2}}{4\left(1+K^{2} / 2\right)}\right]-J_{\frac{n+1}{2}}\left[\frac{n K^{2}}{4\left(1+K^{2} / 2\right)}\right]\right\}^{2} .
\end{aligned}
$$

The factor of 2 in Eq. (14) takes into account that there is no detuning from the on-axis frequency, and hence only one-half of the total flux in the central cone contributes. Figure 3 shows that Eq. (14) is quite accurate for low harmonics but overestimates the brightness by a factor of 2 at higher harmonics, effectively because it does not take into account the rapid variation in intensity with horizontal angle, which in the dipole series model is contained in the $\sin (\Delta)$ term.

In order to include the effect of electron beam emittance we make use of the "addition theorem" or "brightness convolution theorem" introduced by Kim [15] and convolute the brightness function with the electron beam density distribution. We again assume uncorrelated Gaussian distributions $P$ for the electron beam in each phase space coordinate. The central brightness can therefore be written as follows:

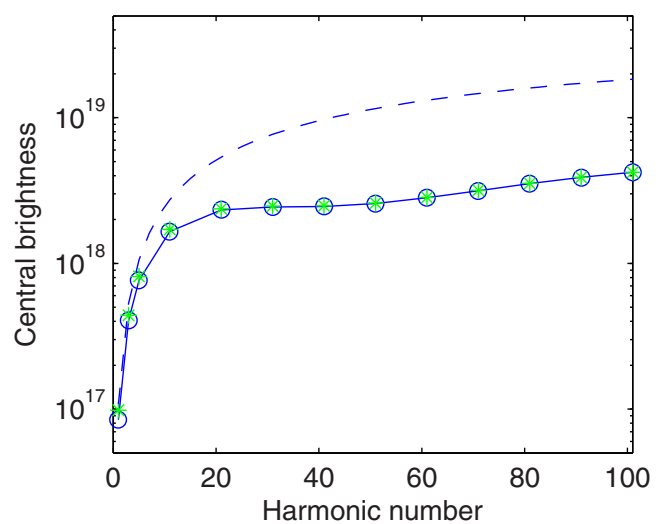

FIG. 4. Central brightness, photons/(s $\mathrm{mm}^{2} \mathrm{mrad}^{2} 0.1 \%$ bandwidth), as a function of harmonic number, with nonzero emittance, zero energy spread; blue circles-Eq. (15); green asterisks —results obtained using SPECTRA; dashed line-Eq. (16).

$$
\begin{aligned}
\mathscr{B}_{o}= & \frac{2 \varepsilon_{o} c}{h} \frac{1}{\lambda^{2}} \frac{I}{e} \int_{-\infty}^{\infty} \int_{-\infty}^{\infty} \int_{-\infty}^{\infty} \int_{-\infty}^{\infty} P\left(x_{o}\right) P\left(x_{o}^{\prime}\right) P\left(y_{o}\right) \\
& \times P\left(y_{o}^{\prime}\right) d x_{o} d x_{o}^{\prime} d y_{o} d y_{o}^{\prime} \int_{-\infty}^{\infty} \int_{-\infty}^{\infty} E_{x}^{*}\left(x_{o}^{\prime}+\frac{\theta_{x}}{2}, y_{o}^{\prime}+\frac{\theta_{y}}{2}\right) \\
& \times E_{x}\left(x_{o}^{\prime}-\frac{\theta_{x}}{2}, y_{o}^{\prime}-\frac{\theta_{y}}{2}\right) \\
& \times \exp \left(-i \frac{2 \pi}{\lambda}\left(x_{o} \theta_{x}+y_{o} \theta_{y}\right)\right) d \theta_{x} d \theta_{y} .
\end{aligned}
$$

The integrations over $x_{o}$ and $y_{o}$ can be carried out using a standard integral, and after simplifying we obtain

$$
\begin{aligned}
\mathscr{B}_{o}= & \frac{2 \varepsilon_{o} c}{h} \frac{4}{\lambda^{2}} \frac{I}{e} \int_{-\infty}^{\infty} \int_{-\infty}^{\infty} \int_{-\infty}^{\infty} \int_{-\infty}^{\infty} P\left(x_{o}^{\prime}\right) P\left(y_{o}^{\prime}\right) \\
& \times P\left(\theta_{x}\right) P\left(\theta_{y}\right) d \theta_{x} d \theta_{y} d x_{o}^{\prime} d y_{o}^{\prime} \\
& \times E_{x}^{*}\left(x_{o}^{\prime}+\theta_{x}, y_{o}^{\prime}+\theta_{y}\right) E_{x}\left(x_{o}^{\prime}-\theta_{x}, y_{o}^{\prime}-\theta_{y}\right),
\end{aligned}
$$

where $P\left(\theta_{x}\right)=\exp -\left(\frac{\theta_{x}^{2}}{2 \sigma_{\theta_{x}}^{2}}\right)$ with $\sigma_{\theta_{x}}=\frac{\lambda}{4 \pi \sigma_{x}}$, and similarly for the $y$-plane.

Figure 4 shows the equivalent plot to Fig. 3 with electron beam emittance included. Again the results are in good agreement with those obtained with SPECTRA. We also compare these results with the commonly used approximate expression for brightness, e.g. [18]:

$$
\mathscr{B}_{o}=\frac{\Phi_{n} / 2}{4 \pi^{2}\left(\sigma_{x}^{2}+\sigma_{R}^{2}\right)^{1 / 2}\left(\sigma_{y}^{2}+\sigma_{R}^{2}\right)^{1 / 2}\left(\sigma_{x^{\prime}}^{2}+\sigma_{R^{\prime}}^{2}\right)^{1 / 2}\left(\sigma_{y^{\prime}}^{2}+\sigma_{R^{\prime}}^{2}\right)^{1 / 2}}
$$


where the rms photon beam size and divergence are given by $\sigma_{R^{\prime}}=\sqrt{\lambda / 2 L}$ and $\sigma_{R}=\sqrt{\lambda L / 8 \pi^{2}}$ respectively. Again it can be seen that for low harmonic numbers there is good agreement between numerical simulations and the simple formula above, but at large $n$ there is now a factor of 4 difference, which is again due to the additional interference effects in the horizontal plane and the fact that there is now a double integration over horizontal angle.

Finally we will also include in some calculations the effect of electron beam energy spread, again by summing the brightness of individual electrons:

$$
\mathscr{B}_{o}=\int_{-\infty}^{\infty} \mathscr{B}_{o}(\varepsilon) P(\varepsilon) d \varepsilon,
$$

where $\varepsilon$ is the relative energy deviation, with Gaussian probability distribution given by $P(\varepsilon)=\exp -\left(\varepsilon^{2} / 2 \sigma_{\varepsilon}^{2}\right) /$ $\sqrt{2 \pi} \sigma_{\varepsilon}$.

\section{COMPARISON OF THE GEOMETRICAL OPTICS AND WAVE OPTICS MODELS}

\section{A. Introduction}

In examining the validity of the geometrical optics model it should be remembered that it implicitly assumes a continuous and smooth distribution of intensity as a function of horizontal angle, or in other words that the interference effects, which are present in any periodic magnet such as an undulator or wiggler, are "smoothed out" in some way. Smoothing can occur for a variety of reasons such as the effects of electron beam emittance and energy spread, the angular acceptance and the degree of monochromaticity of the radiation, in relation to the harmonic number. We will now proceed to make detailed comparisons between the geometrical optics and wave optics methods of calculation, first regarding the spatial distribution in the horizontal plane, and then the radiation brightness, using unless otherwise stated the same case examined in Ref. [24], see Table I.

\section{B. Source size}

Figure 5 shows the flux density distribution in the horizontal plane calculated using the simplified wave optics model described above and it can be seen that it agrees remarkably well with the results shown in Fig. 15 of Ref. [24].

Figure 6 shows results obtained using the geometrical optics calculation. It can be seen that the broadening of the source size as the horizontal acceptance angle increases is much greater than the true result when calculated using Eq. (5) (upper plot), however, as noted above, this is not comparing the same situation. When instead the calculation is performed correctly taking a horizontal cut of the image at $y=0$ (lower plot) the result is now much more similar to that of Fig. 5.

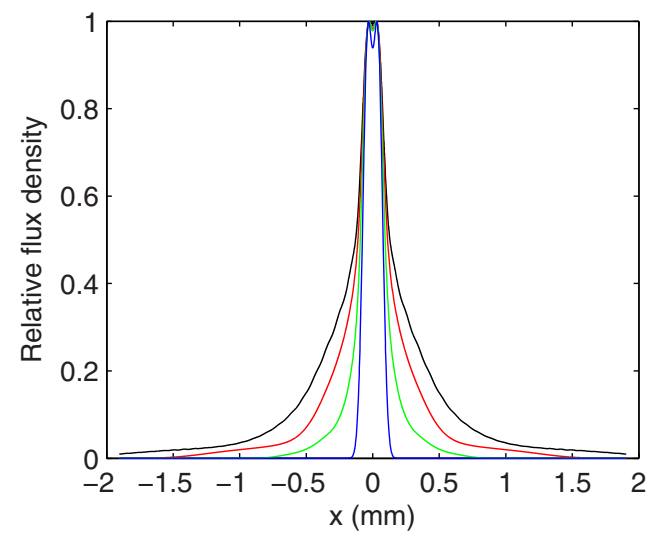

FIG. 5. Spatial flux density (relative units) in the center of the wiggler, horizontal cut at $y=0$, calculated with the simplified wave optics mode, Eqs. (11) and (12), with zero energy spread and various aperture sizes: $\pm 0.125 \mathrm{mrad}$ (blue), $\pm 0.5 \mathrm{mrad}$ (green), $\pm 1.0 \mathrm{mrad}$ (red), $\pm 1.5 \mathrm{mrad}$ (black); vertical aperture: \pm 0.05 mrad.

To compare the models in more detail, Fig. 7 shows the numerically calculated rms and FWHM values as a function of horizontal acceptance. The agreement of the correct geometrical correct optics calculation, Eq. (8), with
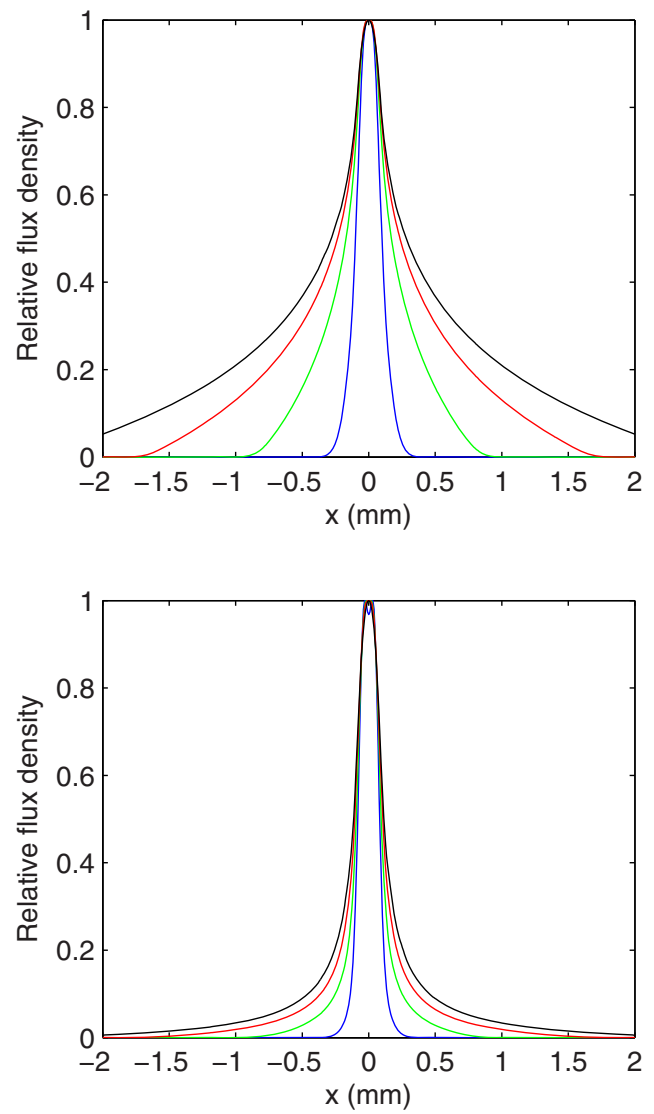

FIG. 6. As Fig. 5, calculated using the geometrical optics model; upper-integrated vertically, Eq. (5); lower-horizontal cut at $y=0$, Eq. (8). 


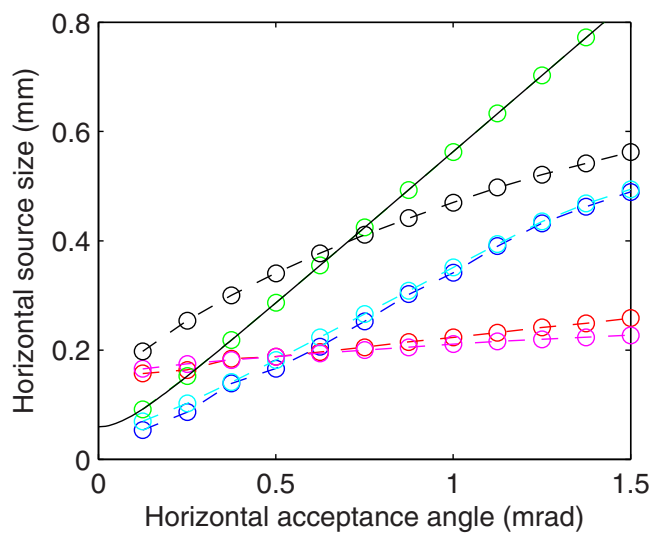

FIG. 7. Horizontal source size as a function of horizontal acceptance angle with zero energy spread, using various calculation methods. Blue (rms), red (FWHM) — simplified wave optics model; cyan (rms), magenta (FWHM) — geometrical optics model, horizontal cut at $y=0$, Eq. (8); green (rms), black (FWHM) - geometrical optics model, integrated over $y$, Eq. (5); dashed lines guide the eye, solid black line-Eq. (7).

the wave optics model is very good, whereas, as seen above, Eq. (5) greatly exaggerates the source widening.

\section{Effect of electron beam divergence}

The authors of Ref. [24] maintain that "the image of the source is insensitive to the electron beam divergence" and they show an example (Fig. 14) where a factor of 2 increase in horizontal beam divergence has a negligible effect. They point out that the brightness function is convoluted with the electron beam distributions at the center of the wiggler and hence there can be no dependence on the length of the device. This is true in principle, since as can be seen from the expression for the spatial distribution for the electric field,

$$
\begin{aligned}
E_{x}(x, y)= & \frac{1}{\lambda} \int E_{x}\left(\theta_{x}, \theta_{y}\right) \\
& \times \exp \left(i \frac{2 \pi}{\lambda}\left[\left(\theta_{x}-x_{o}^{\prime}\right) x+\left(\theta_{y}-y_{o}^{\prime}\right) y\right]\right) d \theta_{x} d \theta_{y},
\end{aligned}
$$

the effect of the electron's angle $x_{o}^{\prime}, y_{o}^{\prime}$ is only to introduce a phase factor which disappears when forming the intensity. However, this proposition is only true if there are no limits to the integration. Equation (11) shows that the effect of an angular aperture introduces a dependence of the source distribution on the beam divergence. In other words, since angular acceptance has an effect on the source size (as shown in Fig. 5 and Ref. [24]), there must equally be a similar effect due to electron beam divergence.

Figure 8 reproduces the example of Ref. [24] using the simplified wave optics and geometrical optics models. In both cases the effect of an increase in horizontal divergence from $31.6 \mu \mathrm{rad}$ (blue curves) to $63.2 \mu \mathrm{rad}$ (red curves) is negligible, as found in Ref. [24], however this is not surprising since the horizontal aperture in this case is
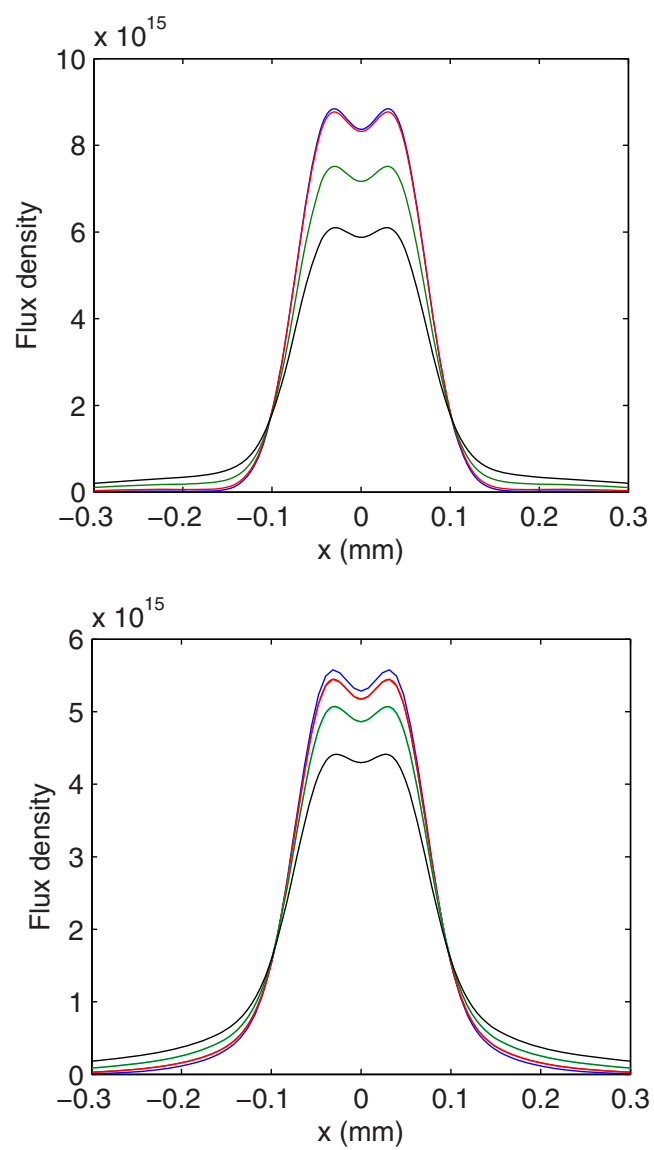

FIG. 8. Spatial flux density, photons/(s $\mathrm{mm}^{2} 0.1 \%$ bandwidth), in the center of the wiggler, horizontal cut at $y=0$, with zero energy spread; blue $-\varepsilon_{x}=1 \mathrm{~nm} \mathrm{rad}, \beta_{x}=1 \mathrm{~m}$; red- $\varepsilon_{x}=$ $2 \mathrm{~nm} \mathrm{rad}, \quad \beta_{x}=0.5 \mathrm{~m} ;$ green $-\varepsilon_{x}=4 \mathrm{~nm} \mathrm{rad}, \quad \beta_{x}=0.25 \mathrm{~m}$; black- $\varepsilon_{x}=8 \mathrm{~nm} \mathrm{rad}, \beta_{x}=0.125 \mathrm{~m}$; upper-simplified wave optics calculation, Eqs. (11) and (12), lower-geometrical optics model, Eq. (8).

$\pm 125 \mu \mathrm{rad}$ and so dominates over the electron beam divergence. Figure 8 shows that increasing the divergence further does produce a noticeable effect which is consistent between the two models. Figure 9 examines this more closely and shows the calculated spatial distribution for two different electron beam divergences, excluding the electron source size. The larger divergence clearly results in a broadening of the distribution.

Figure 10 shows a further comparison of the geometrical optics model with a wave-optics calculation, in this case for a 3T multipole wiggler in NSLS-II, which is reported in Ref. [21]. The plot shows the horizontal cut of the intensity distribution of a focused image of the source for different viewing angles. The agreement with Fig. 4 of Ref. [21] is remarkable, both qualitatively and quantitatively. The authors of Ref. [21] comment that the "partially-coherent simulations reveal a number of features-deviations from Gaussian shapes-in the resulting intensity distributions, especially at the off-axis locations of the collecting aperture," however it is clear from this comparison that these 


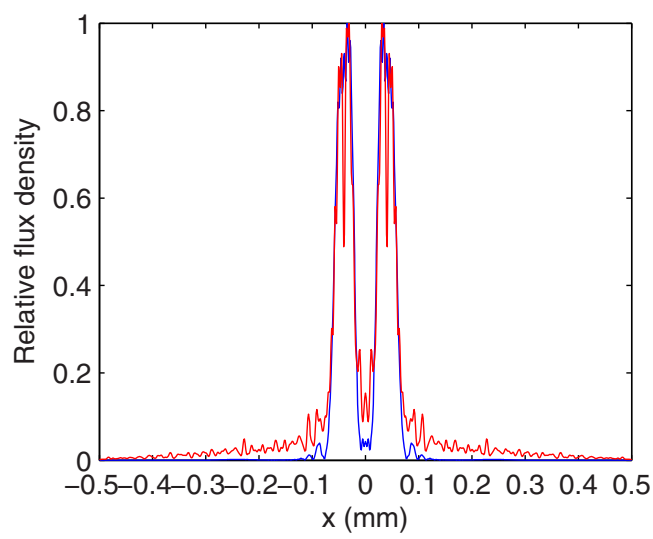

FIG. 9. Spatial flux density (relative units) in the center of the wiggler, horizontal cut at $y=0$, with zero energy spread, zero electron beam size; blue $-\varepsilon_{x}=1 \mathrm{~nm} \mathrm{rad}, \beta_{x}=1 \mathrm{~m}$; red $-\varepsilon_{x}=8 \mathrm{~nm} \mathrm{rad}, \beta_{x}=0.125 \mathrm{~m}$, calculated with the simplified wave optics model, Eq. (11).

features have nothing to do with coherence or interference of waves, but in fact are entirely consistent with a geometrical optics model based on incoherent addition of intensities.

\section{Brightness}

We turn now to a comparison of brightness calculated with the wave optics (i.e. Wigner function) and geometrical optics methods. Given that the underlying assumption of the geometrical optics model is a "smoothing out" of harmonics, we clearly need to include multiple harmonics in the wave optics calculation. This is very demanding of computer time, hence the need for the simplication of the

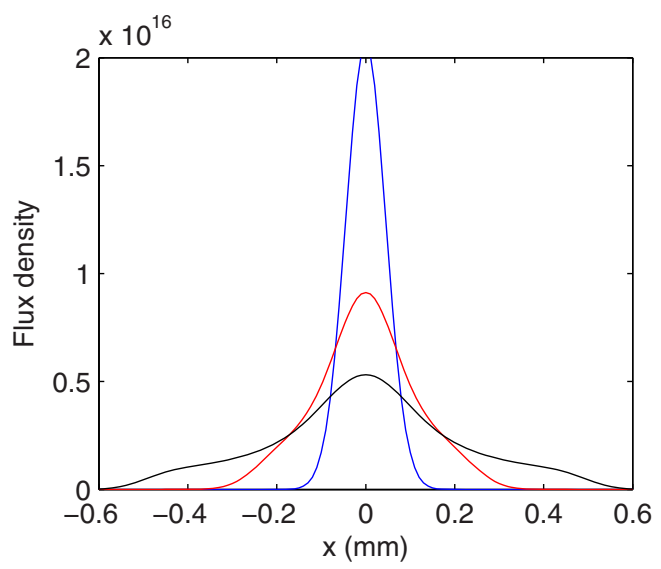

FIG. 10. Spatial flux density, photons $/\left(\mathrm{s} \mathrm{mm}^{2} 0.1 \%\right.$ bandwidth), of $10 \mathrm{keV}$ radiation in the center of a $3 \mathrm{~T}$ wiggler (period $=40 \mathrm{~mm}$, number of poles $=50)$ in NSLS-II $(3 \mathrm{GeV}$, $\left.0.5 \mathrm{~A}, \varepsilon_{x}=0.9 \mathrm{~nm}, \varepsilon_{y}=8 \mathrm{pm}, \beta_{x}=2 \mathrm{~m}, \beta_{y}=1 \mathrm{~m}\right)$, as in Ref. [21]. Angular acceptance $\pm 0.05 \mathrm{mrad}$ horizontal, $\pm 0.05 \mathrm{mrad}$ vertical, at $0 \mathrm{mrad}$ (blue), $0.5 \mathrm{mrad}$ (red) and $1.0 \mathrm{mrad}$ (black) off-axis horizontally, horizontal cut at $y=0$, calculated with the geometrical optics model, Eq. (8). problem described in Sec. III B above. We also need to be in a regime where multiple harmonics can contribute. The parameters chosen in Ref. [24] for simulation are not ones where a smearing of harmonics is expected since both the electron beam divergence and harmonic number are too small to exhibit these effects.

Based on the angular separation between the $n+1$ th harmonic off axis and the $n$th harmonic on axis, at the same photon energy, we might expect smearing when

$$
\gamma \sigma_{x^{\prime}, y^{\prime}}>\sqrt{\left(1+K^{2} / 2\right) / n}
$$

In the present case with $n=101$ this would require $\sigma_{x^{\prime}}$ to be $148 \mu \mathrm{rad}$, which is much larger than the actual value of $32 \mu \mathrm{rad}$ (Table I and Ref. [24]). However, the photon energies of interest for a wiggler source are usually much greater than the 101st harmonic. Such sources are generally used around the critical energy, which in this case is close to the 701st harmonic.

Figure 11 shows the calculated central brightness as a function of horizontal emittance for the 701st harmonic, including results when the angular ranges in Eq. (15) are restricted such that only one harmonic is taken into consideration, which agree with the SPECTRA results, and also when the full integration range is included, covering many harmonics (which is not possible with SPECTRA [28]). When the emittance is small only the main harmonic contributes and as expected the brightness exceeds that calculated by the geometrical optics model. However, as the emittance increases, the single harmonic result falls below that given by the geometrical optics model, but the result obtained by integrating over many harmonics is in very good agreement with it. We note also that the brightness obtained from the simple formula Eq. (4) is

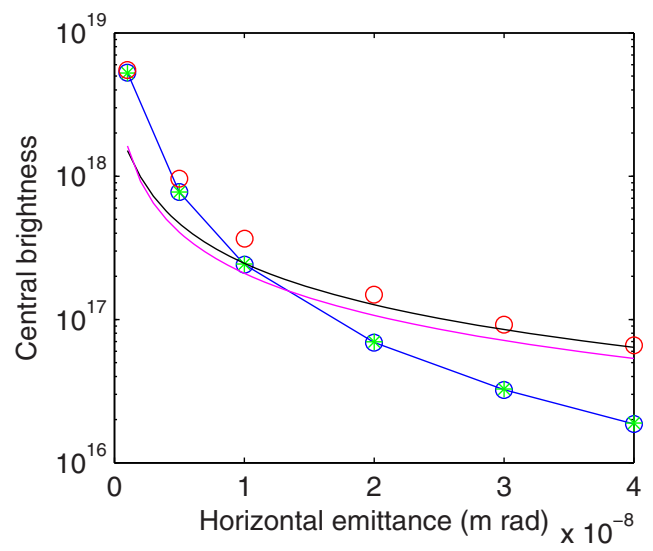

FIG. 11. Central brightness, photons/(s $\mathrm{mm}^{2} \operatorname{mrad}^{2} 0.1 \%$ bandwidth), for the 701st harmonic as a function of horizontal emittance, $\varepsilon_{y} / \varepsilon_{x}=1 \%$, zero energy spread. Blue circles-single harmonic; green asterisks - single harmonic using SPECTRA; red circles-multiple harmonics; black line-Eq. (3), magenta line-Eq. (4). 


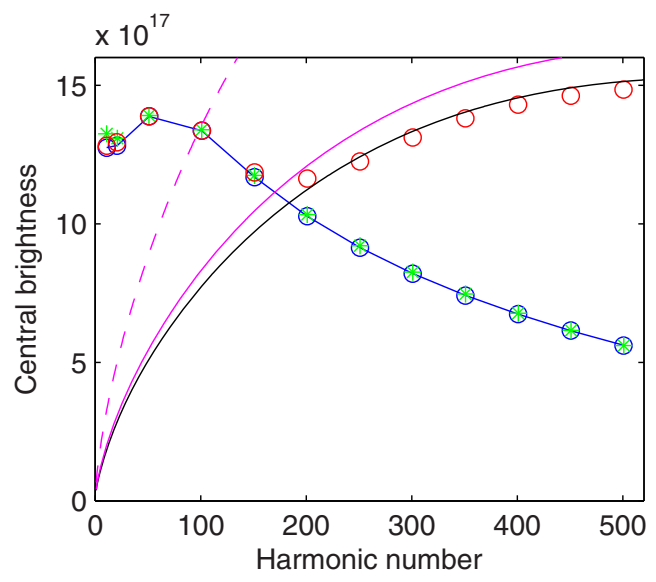

FIG. 12. Central brightness, photons/(s $\mathrm{mm}^{2} \operatorname{mrad}^{2} 0.1 \%$ bandwidth), as a function of harmonic number, including emittance and energy spread. Blue circles-single harmonic; green asterisks - single harmonic using SPECTRA; red circlesmultiple harmonics; black line-Eq. (3), magenta line-Eq. (4), dashed magenta line-Eq. (4) with terms in $L$ set to zero.

also in good agreement with these results. It is clear therefore that the wave optics model for brightness does agree with the simple geometrical model when the conditions are such to allow a smearing out of the harmonics.

As further proof of this fact, we now introduce another very important smearing mechanism which is ignored in the calculations of Ref. [24], namely energy spread. We expect a smearing of harmoncs due to energy spread roughly when the following condition is satisfied:

$$
\sigma_{\varepsilon}>\frac{1}{4 n}
$$

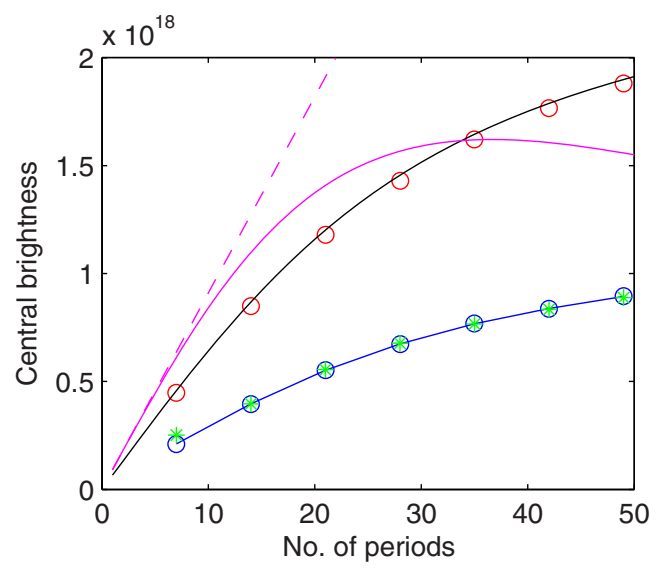

FIG. 13. Central brightness, photons $/\left(\mathrm{s} \mathrm{mm}^{2} \operatorname{mrad}^{2} 0.1 \%\right.$ bandwidth), as a function of number of wiggler periods, $n=401$, including emittance and energy spread. Blue circles — single harmonic; green asterisks - single harmonic using SPECTRA; red circles-multiple harmonics; black line-Eq. (3), magenta line-Eq. (4), dashed magenta line-Eq. (4) with terms in $L$ set to zero.

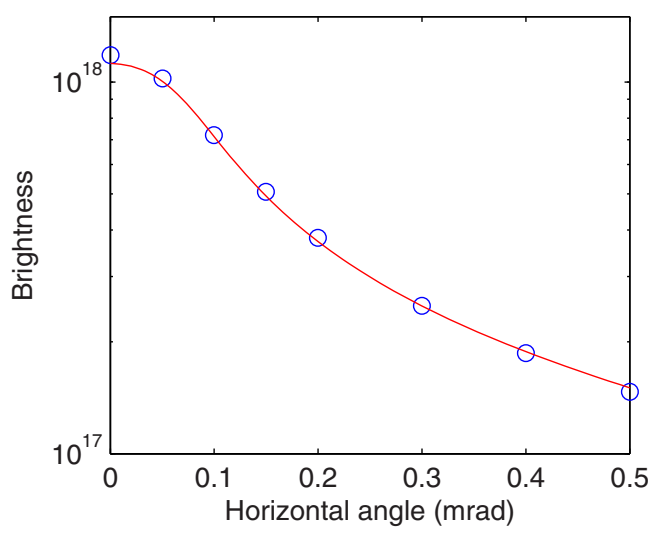

FIG. 14. Brightness, photons/(s $\mathrm{mm}^{2} \operatorname{mrad}^{2} 0.1 \%$ bandwidth), as a function of angle off-axis in the horizontal plane, $n=201$, including emittance and energy spread; blue circles-simplified wave optics model with multiple harmonics; red linegeometrical optics Eq. (2).

which in the present case $\left(\sigma_{\varepsilon}=0.001\right)$ would suggest $n>250$. Figure 12 shows the calculated central brightness as a function of harmonic number, in the same format as Fig. 11, now including both emittance and energy spread. Again the single harmonic calculation agrees with the result from SPECTRA, and at low harmonic numbers a single harmonic dominates. At larger harmonic nunbers, roughly in agreement with the criterion above, the result obtained with multiple harmonics begins to deviate and at the same time align more cosely with the result of the geometrical optics model. The approximation for the geometrical model is also in reasonable agreement with this, and it is clear that if the terms in $L$ are ignored that the agreement is very poor. Thus again it is clear that the wave optics calculation tends to that of the geometrical optics calculation when smoothing of the harmonics takes place, in this case due to energy spread.

Figure 13 shows the central brightness for $n=401$ as a function of the number of wiggler periods, including both emittance and energy spread. Again there is good agreement between the wave optics calculation, when integrating over multiple harmonics, with the geometrical optics model. The approximation Eq. (4) agrees reasonably well for small number of periods, but as expected deviates when the wiggler length becomes too great. Once again, ignoring the depth-of-field terms in Eq. (4) makes the agreement much worse.

Finally, Fig. 14 shows the brightness as a function of the off-axis angle in the horizontal plane for the 201st harmonic and there is once again impressive agreement with the geometrical optics model.

\section{DISCUSSION}

The above results clearly show that there is a depth-offield broadening of the horizontal source size as a function of acceptance angle, and hence also angular divergence, in 
both the wave optics and geometrical optics models. The question then arises as to where these effects come from in the wave optics model, when there is no obvious $\theta_{x} L$ dependence, as in the case of geometric projection. The answer lies in the line shape factor in the equation for the electric field, Eq. (9), and how this relates to the spatial distribution in the center of the wiggler, which is the Fourier transform of the far-field radiation pattern Eq. (10). Near the harmonic which occurs off axis at angle $\theta_{x}$ (at the photon energy determined by the $n$th harmonic on axis) the line shape factor can be written as follows:

$$
\frac{\sin \left(N \pi \omega / \omega_{1}\right)}{\sin \left(\pi \omega / \omega_{1}\right)} \approx N \operatorname{sinc}(N \pi \delta),
$$

where $\delta$ can be written in angular terms as

$$
\delta=2 n \frac{\gamma^{2}}{1+K^{2} / 2} \theta_{x} \Delta \theta_{x}
$$

and so the line shape factor becomes $N \operatorname{sinc}\left(\pi \theta_{x} L \frac{\Delta \theta_{x}}{\lambda}\right)$.

The transform of a sinc-function with first zero at $\Delta \theta_{x} / \lambda=1 / \theta_{x} L$ is a top-hat function of width $x=$ $\pm \theta_{x} L / 2$ [29], and so we have recovered the result that would be expected from simple geometrical considerations. This explains, approximately, the extent of the spatial distribution in both Fig. 5, where the broadening is dominated by the large acceptance angles, and Fig. 9 (red curve), which is dominated by the large beam divergence.

Concerning the calculation of brightness, an aspect worth commenting on is the fact that only when the integration is carried out over the full angular range in Eq. (15), allowing multiple harmonics to contribute, do the results agree with the geometrical optics model. On the one hand, this seems quite natural as we are expecting harmonics to become "smeared out," and indeed multiple harmonics are also included in the calculation of source size, Eq. (11), but in the case of brightness it raises an interesting point with regard to the validity of the Wigner formulation as representing brightness. It is well known that there are some difficulties with this definition, see for example Refs. [15,18,20,23], most notably because the brightness so defined can be negative, for example for even harmonics. Despite this conceptual difficulty however, it appears that the significant negative contributions to the overall brightness are actually essential to achieving a correct result. To take one example, for the 401st harmonic in Fig. 12, calculations reveal that the positive contributions to the brightness when integrating over the harmonics amount to $1.98 \times 10^{18}$, the negative to $-0.55 \times 10^{18}$, and hence the overall sum is $1.43 \times 10^{18}$ in standard units, photons/(s mm $\mathrm{mrad}^{2} 0.1 \%$ bandwidth), which is in good agreement with the geometrical optics calculation.

\section{CONCLUSION}

The geometrical optics model referred to in [24], namely Eq. (7), was clearly not representative of all aspects of the model. In particular, we have demonstrated here that the spatial distribution in the center of the wiggler changes substantially when a horizontal cut is taken rather than an integral over the vertical position. As a result, there is much less "depth of field broadening" predicted by geometrical optics as a function of horizontal angle or aperture than supposed in Ref. [24]. The incorrect application of the geometrical optics model, and the limited analysis which did not fully test its validity, led the authors of [24] to incorrectly conclude that "depth of field effects do not exist."

On the contrary, we have shown here that the geometrical optics and wave optics methods of calculation give consistent results, both for source size and brightness, provided there is sufficient "smoothing" of the interference structure. In the case of calculating horizontal source size, large acceptance angles can be sufficient to achieve this condition, but for calculating central or peak brightness the models only agree at a sufficiently high harmonic number with either large electron beam divergence or when energy spread is included.

A reconciliation between wave optics and geometrical optics was achieved many years ago in the case of undulator radiation, however it is only relatively recently that the more complex case of wiggler radiation started to be analyzed using a wave optics approach. Despite the extensive body of work which has analyzed wiggler radiation in terms of geometrical optics, and some measurements which are consistent with it, Ref. [24] instead threw doubt on the validity of the geometrical optics calculation. We have shown here however that the two methods are indeed consistent, and so finally it now appears that geometrical and wave optics can be reconciled also in the more complex case of radiation from multipole wigglers.

\section{ACKNOWLEDGMENTS}

It is a pleasure to thank L. Berman for drawing my attention to Ref. [24], encouraging me to look further into the validity of the geometrical optics wiggler model, and for supplying the measurement data for Fig. 1.

[1] A. P. Sabersky, The geometry and optics of synchrotron radiation, Part. Accel. 5, 199 (1973).

[2] G. K. Green, BNL 50522 (1976).

[3] R. Coïsson, S. Guiducci, and M. A. Preger, Multipole wigglers as sources of synchrotron radiation, Nucl. Instrum. Methods Phys. Res. 201, 3 (1982).

[4] K. J. Kim, Brightness and coherence of synchrotron radiation and high-gain free electron lasers, Nucl. Instrum. Methods Phys. Res., Sect. A 261, 44 (1987). 
[5] R. P. Walker, Sincrotrone Trieste Report No. ST/M-TN-89/ 24, 1988.

[6] G. E. van Dorssen, H. A. Padmore, and W. Joho, Optimization and comparison of multipole wiggler and bending magnet sources of synchrotron radiation for x-ray experiments, Proc. SPIE Int. Soc. Opt. Eng. 2013, 104 (1993).

[7] H. A. Padmore, T. Earnest, S.-H. Kim, A. C. Thompson, and A.L. Robinson, A beamline for macromolecular crystallography at the Advanced Light Source, Rev. Sci. Instrum. 66, 1738 (1995).

[8] R. P. Walker, Bending magnet and wiggler radiation, in Undulators, Wigglers, and their Applications, edited by H. Onuki and P. Elleaume (Taylor \& Francis, London, 2003), Chap. 4.

[9] H. Amenitsch, S. Bernstorf,, M. Kriechbaum, D. Lombardo, H. Mio, M. Rappolt, and P. Laggner, Performance and first results of the ELETTRA high-flux beamline for small-angle X-ray scattering, J. Appl. Crystallogr. 30, 872 (1997).

[10] L. E. Berman and Z. Yin, Off-axis viewing of radiation emission by long wiggler sources, Nucl. Instrum. Methods Phys. Res., Sect. A 649, 35 (2011).

[11] D. F. Alferov, Y. A. Bashmakov and E. G. Bessonov, Undulator radiation, Sov. Phys. Tech. Phys. 18, 1336 (1974).

[12] B. M. Kincaid, A short-period helical wiggler as an improved source of synchrotron radiation, J. Appl. Phys. 48, 2684 (1977).

[13] G. Brown, K. Halbach, J. Harris, and H. Winick, Wiggler and undulator magnets - A review, Nucl. Instrum. Methods Phys. Res. 208, 65 (1983).

[14] S. Krinsky, Undulators as sources of synchrotron radiation, IEEE Trans. Nucl. Sci. 30, 3078 (1983).

[15] K. J. Kim, Brightness, coherence and propagation characteristics of synchrotron radiation, Nucl. Instrum. Methods Phys. Res., Sect. A 246, 71 (1986); A new formulation of synchrotron radiation optics using the wigner distribution, Proc. SPIE Int. Soc. Opt. Eng. 582, 2 (1986).
[16] R. Coisson and R. P. Walker, Phase space distribution of brilliance of undulator sources, Proc. SPIE Int. Soc. Opt. Eng. 582, 24 (1986).

[17] J. Bahrdt, Wave-front propagation: design code for synchrotron radiation beam lines, Appl. Opt. 36, 4367 (1997).

[18] P. Elleaume, Synchrotron radiation, generalities, in Undulators, Wigglers, and their Applications (Ref. [8]), Chap. 2; Undulator radiation, in Undulators, Wigglers, and their Applications (Ref. [8]), Chap. 3.

[19] T. Tanaka and H. Kitamura, Recent progress of the synchrotron radiation calculation code SPECTRA, AIP Conf. Proc. 879, 355 (2007).

[20] I. V. Bazarov, Synchrotron radiation representation in phase space, Phys. Rev. ST Accel. Beams 15, 050703 (2012).

[21] O. Chubar, A. Fluerasu, L. Berman, K. Kaznatcheev, and L. Wiegart, Wavefront propagation simulations for beamlines and experiments with "Synchrotron Radiation Workshop”, J. Phys. Conf. Ser. 425, 162001 (2013).

[22] T. Tanaka, Numerical methods for characterization of synchrotron radiation based on the Wigner function method, Phys. Rev. ST Accel. Beams 17, 060702 (2014).

[23] G. Geloni, V. Kocharyan, and E. Saldin, Brightness of synchrotron radiation from undulators and bending magnets, J. Synchrotron Radiat. 22, 288 (2015).

[24] G. Geloni, V. Kocharyan, and E. Saldin, Brightness of synchrotron radiation from wigglers, Nucl. Instrum. Methods Phys. Res., Sect. A 807, 13 (2016).

[25] R. P. Walker, Interference effects in undulator and wiggler radiation sources, Nucl. Instrum. Methods Phys. Res., Sect. A 335, 328 (1993).

[26] R. P. Walker, Phase errors and their effect on undulator radiation properties, Phys. Rev. ST Accel. Beams 16, 010704 (2013).

[27] SPECTRA v. 10.0.7 available from http://radiant.harima .riken.go.jp/spectra/index.html.

[28] T. Tanaka (private communication).

[29] J. F. James, A Student's Guide to Fourier Transforms, with Applications in Physics and Engineering, 3rd ed. (Cambridge University Press, Cambridge, 2011). 